\title{
Design and Synthesis of Activity Probes for Glycosidases
}

\author{
Charng-Sheng Tsai, ${ }^{\dagger}$ Yaw-Kuen Li, ${ }^{\ddagger}$ and Lee-Chiang Lo ${ }^{*, \dagger}$ \\ Department of Chemistry, National Taiwan University, Taipei 106, Taiwan, \\ and Department of Applied Chemistry, The National Chiao-Tung University, \\ Hsin-Chu 300, Taiwan
}

lclo@ccms.ntu.edu.tw

Received July 16, 2002

ABSTRACT

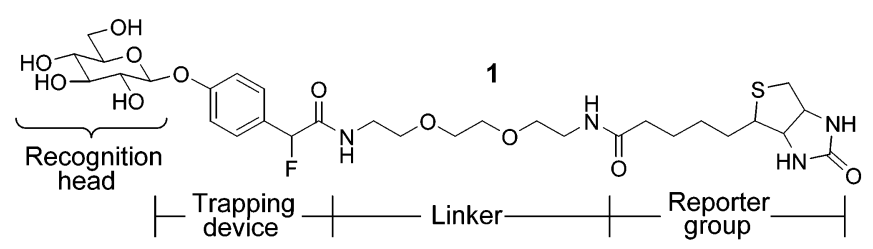

A new synthetic route was developed for the preparation of activity probe 1 for $\beta$-glucosidase in this study. The key glycosidation step begins with benzyl $p$-hydroxyphenylacetate. Benzylic functionalization for the construction of the trapping device was achieved at later stages. Probe 1 was shown to be able to label the target enzyme. This cassette-like design offers great flexibility for future alterations. It would allow the synthetic scheme to expand to other glycosidase probes with different linker/reporter combinations.

Activity probes have been developed and found to be useful in the mechanism-based labeling and selection of biocatalysts with designated hydrolytic activities, including mutant $\beta$ lactamases $^{1}$ and catalytic antibodies, ${ }^{2}$ from libraries. More recently, these class-selective probes have been demonstrated to be a powerful tool in proteomics studies for identifying certain hydrolase families such as protein tyrosine phosphatases $^{3,4}$ and serine hydrolases. ${ }^{5-8}$ They helped simplify the otherwise complicated protein band/spot patterns after gel electrophoresis. Protein phosphorylation and glycosylation are two mechanisms commonly seen in biological

\footnotetext{
National Taiwan University.

$\doteqdot$ The National Chiao-Tung University.

(1) Soumillion, P.; Jespers, L.; Bouchet, M.; Marchand-Brynaert, J.; Winter, G.; Fastrez, J. J. Mol. Biol. 1994, 237, 415-422.

(2) Janda, K. D.; Lo, L.-C.; Lo, C.-H. L.; Sim, M.-M.; Wang, R.; Wong,

C.-H.; Lerner, R. A. Science 1997, 275, 945-948.

(3) Lo, L.-C.; Wang, H.-Y.; Wang, Z.-J. J. Chin. Chem. Soc. 1999, 46, $715-718$.

(4) Lo, L.-C.; Pang, T.-L.; Kuo, C.-H.; Chiang, Y.-L.; Wang, H.-Y.; Lin, J.-J. J. Proteome Res. 2002, 1, 35-40.

(5) Liu, Y.; Patricelli, M. P.; Cravatt, B. F. Proc. Natl. Acad. Sci. U.S.A. 1999, 96, 14694-14699. 668.

(6) Cravatt, B. F.; Sorensen, E. J. Curr. Opin. Chem. Biol. 2000, 4, 663-

(7) Kidd, D., Liu, Y., Cravatt, B. F. Biochemistry 2001, 40, 4005-4015.

(8) Patricelli, M. P.; Giang, D. K.; Stamp, L. M.; Burbaum, J. J.
} Proteomics 2001, 1, 1067-1071. systems for regulating protein activities. The major hydrolytic enzymes involved in these processes are phosphatases and various glycosidases. Therefore, these two enzyme families are often important targets in biomedical studies. Since we have previously reported the probes for protein tyrosine phosphatases adopting a cassette-like design,, 3 we now extend the target activities to glycosidase families. The trapping device, which makes use of the quinone methide chemistry $^{9}$ and is the core of the probe, was derived from a $p$-hydroxymandelic acid derivative. ${ }^{10}$ However, due to difficulties in the glycosidation step with the $p$-hydroxymandelic acid derivative, in this study we explored a new synthetic route for the preparation of activity probe $\mathbf{1}$ and studied it on a model $\beta$-glucosidase.

Activity probe 1 thus designed carries a glucose residue serving as the recognition head. This recognition head is connected through a $\beta$-glycosidic linkage to a $p$-hydroxybenzylic fluoride moiety. When the designated glycosidic bond is cleaved by a $\beta$-glucosidase, it undergoes $1,6-$ elimination to remove a fluoride and generate a reactive quinone methide. This reactive quinone methide intermediate

(9) Wakselman, M. Nouv. J. Chim. 1983, 7, 439-447.

(10) Lo, L.-C.; Lo, C.-H. L.; Janda, K. D.; Kassel, D. B.; Raushel, F. M. Bioorg. Med. Chem. Lett. 1996, 6, 2117-2120. 


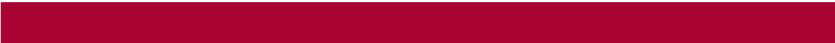

Scheme 1

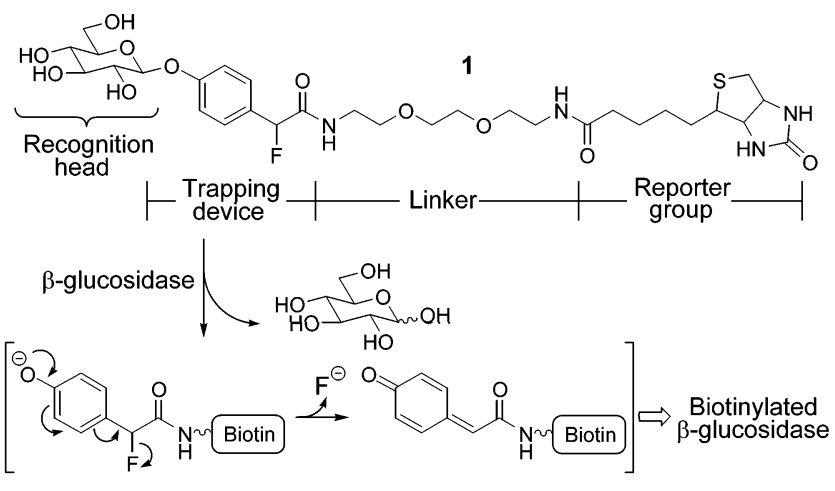

serves as the trapping device to alkylate nearby nucleophiles on the biocatalysts, resulting in the biotinylated enzyme (Scheme 1). The biotin reporter group in probe $\mathbf{1}$ not only is useful for highly sensitive secondary detection but also offers the advantage of enriching the labeled proteins in proteomic studies.

The synthesis of probe $\mathbf{1}$ begins with benzyl $p$-hydroxyphenylacetate $\mathbf{3}$ (Scheme 2). It was reacted with glucosyl-

Scheme $2^{a}$

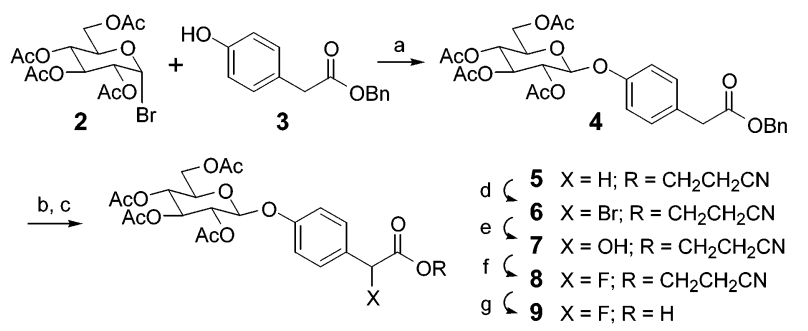

a (a) AgOTf, $\mathrm{CH}_{2} \mathrm{Cl}_{2}$ (67\%); (b) $\mathrm{H}_{2}, \mathrm{Pd} / \mathrm{C}$ (91\%); (c) DCC, DMAP, $\mathrm{HOCH}_{2} \mathrm{CH}_{2} \mathrm{CN}, \mathrm{CH}_{2} \mathrm{Cl}_{2}$ (95\%); (d) NBS, $\mathrm{CCl}_{4}, h v(92 \%$ ); (e) $\mathrm{AgNO}_{3}$, acetone/ $\mathrm{H}_{2} \mathrm{O}$ (55\%); (f) DAST, $\mathrm{CH}_{2} \mathrm{Cl}_{2}$ (85\%); (g) $\mathrm{DBU}, \mathrm{CH}_{2} \mathrm{Cl}_{2}(95 \%)$.

bromide 2 in the presence of AgOTf to give $\beta$-glucoside 4 in $67 \%$ yield. The $\beta$-configuration of the glycosidic linkage was indicated by the coupling constant between $\mathrm{H}-1$ and $\mathrm{H}-2$ $(J=7.6 \mathrm{~Hz})$. The conversion of the benzyl protecting group in compound 4 to a $\beta$-cyanoethyl group was achieved by hydrogenation followed by coupling with DCC/DMAP/ $\mathrm{HOCH}_{2} \mathrm{CH}_{2} \mathrm{CN}$ to give $\beta$-cyanoethyl ester 5 in $86 \%$ yield for two steps. The benzylic functionalization was introduced first by bromination with NBS under irradiation, ${ }^{11}$ followed by hydrolysis with $\mathrm{AgNO}_{3}$ in acetone/ $\mathrm{H}_{2} \mathrm{O}$ to give benzylic alcohol intermediate 7 in $51 \%$ yield for two steps. The newly formed hydroxyl group was converted to fluoride by reaction with DAST to offer the protected half unit $\mathbf{8}$, covering the recognition head and the trapping device, in $85 \%$ yield. The

(11) Briggs, J. C.; Haines, A. H.; Taylor, R. J. K. J. Chem. Soc., Perkin Trans. I 1995, 27-32. $\beta$-cyanoethyl group of compound $\mathbf{8}$ was removed by treatment with DBU to form $\alpha$-fluoroacid 9 in good yields (95\%). Since the free acid of compound 9 was not stable, it was stored as its triethylammonium salt.

The complete skeleton of the probe was constructed by amide bond formation between $\alpha$-fluorocarboxylate 9 and the TFA salt of dioxaoctanediamine derivative of biotin $\mathbf{1 0}^{12,13}$ in the presence of DCC/HOBt (Scheme 3). The fully

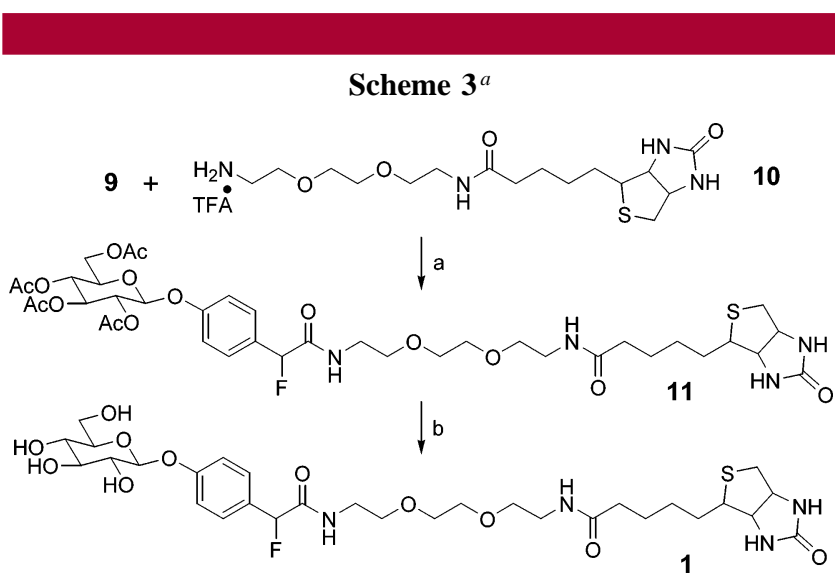

a (a) DCC, HOBt, TEA, DMF (83\%); (b) $\mathrm{Na}_{2} \mathrm{CO}_{3}, \mathrm{MeOH}(92 \%)$.

protected probe $\mathbf{1 1}$ was then subjected to final deacetylation by treatment with $\mathrm{Na}_{2} \mathrm{CO}_{3} / \mathrm{MeOH}$ to give probe $\mathbf{1}$ in high yields.

In the preliminary study, $\beta$-glucosidase cloned from Flavobacterium meningosepticum was employed. ${ }^{14,15}$ This enzyme belongs to the family 3 hydrolases. The catalytic mechanisms of this $\beta$-glucosidase and other family 1 hydrolases have been extensively studied. ${ }^{16,17}$ To demonstrate that probe 1 was a substrate of $\beta$-glucosidase and the concomitant elimination from the hydrolyzed intermediate really occurred, we performed time course experiments by incubating probe $1(6.1 \mathrm{mM})$ with enzyme $(0.66 \mu \mathrm{M})$ in phosphate buffer (50 mM, pH 7.0). The fluoride signals were monitored with ${ }^{19} \mathrm{~F}$ NMR. As shown in Figure 1, at time zero, the two sets of doublets around -168 ppm represent the signals of probe $\mathbf{1}$, which exists as a pair of diastereomers (shown in inset). Both diastereomers of probe $\mathbf{1}$ underwent hydrolysis by the enzyme in a comparable rate to release inorganic fluoride, supporting the 1,6-elimination depicted in Scheme 1. The new singlet at $-122 \mathrm{ppm}$ results from inorganic fluoride. Probe $\mathbf{1}$ did not produce any activation/ elimination in the absence of $\beta$-glucosidase.

(12) Sigal, G. B.; Mammen, M.; Dahmann, G.; Whitesides, G. M. J. Am. Chem. Soc. 1996, 118, 3789-3800.

(13) Braun, M.; Camps, X.; Vostrowsky, O.; Hirsch, A.; Endress, E.; Bayerl, T. M.; Birkert, O.; Gauglitz, G. Eur. J. Org. Chem. 2000, 7, 11731182 .

(14) Li, Y.-K.; Chu, S.-H.; Sung, Y.-H. J. Chin. Chem. Soc. 1998, 45, $603-610$.

(15) Li, Y.-K.; Lee, J.-A. Enzyme Microb. Technol. 1999, 24, 144150.

(16) Kempton, J. B.; Withers, S. G. Biochemistry 1992, 31, 9961-9969. 840. 


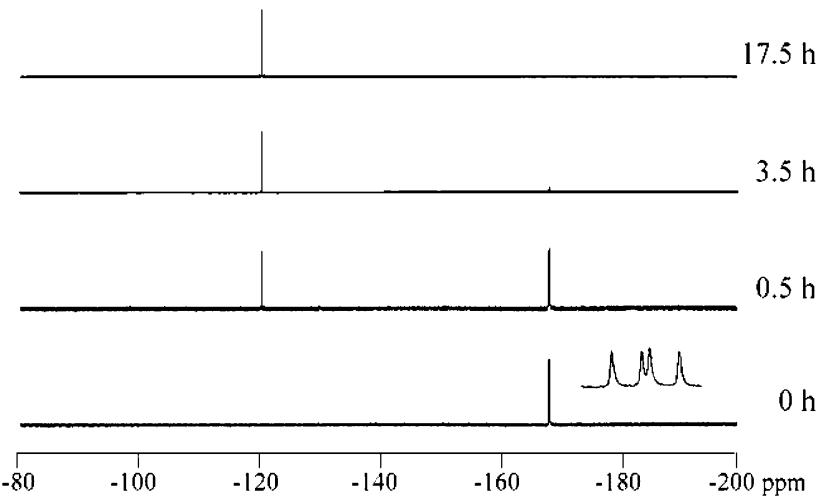

Figure 1. Time course study for the hydrolysis of probe $\mathbf{1}$ by $\beta$-glucosidase as monitored with ${ }^{19} \mathrm{~F}$ NMR at $0,0.5,3.5$, and 17.5 $\mathrm{h}$ : the two sets of doublets (enlarged in inset) at $-168 \mathrm{ppm}$ are the signal for the starting material; the singlet at $-122 \mathrm{ppm}$ represents the inorganic fluoride.

Labeling of the enzyme by activity probe 1 was next examined. Two parallel labeling experiments were carried out and analyzed (Figure 2). On the left-hand side, lanes 1 and 2 show the Coomassie blue stained gel, indicating the relative amounts of the protein loaded. The gel on the right (lanes 3 and 4) was visualized with streptavidin-conjugated peroxidase chemiluminescence after blotting onto a nitrocellulose membrane. It clearly shows the labeling of $\beta$-glucosidase by the probe 1 (lane 4 ). The slightly smeared band with higher molecular weights (lanes 2 and 4) suggests possible multiple modifications. On the contrary, there was no labeling in the absence of probe $\mathbf{1}$ (lane 3). In separate labeling experiments with a higher enzyme concentration $(6.6 \mu \mathrm{M})$, we observed the formation of a protein precipitation within 5 min of mixing. The residual activity was found to be less than $10 \%$ after 30 min of incubation using $p$-nitrophenyl glucoside as the assay substrate. This dramatic activity loss may be attributed to the active site labeling or, more likely, the severe aggregation of the multiply labeled proteins. Although the degree of modification was not determined in this study, the observation of multiple labeling accompanied by the loss of activity is consistent with the previous study on the phosphotriesterase probe bearing an identical trapping device, in which case the multiply labeled proteins were characterized by LC/MS. ${ }^{10}$ A similar phenomenon of multiple labeling by the quinone methide intermediate was also extensively studied on a new mechanism-based inhibitor of $\beta$-glucosidase purified from Agrobacterium faecalis. ${ }^{18}$

It has to be stressed that while suicide substrates based on the generation of quinone methide intermediates have been reported for $\beta$-glucosidases and $\beta$-glucuronidase, ${ }^{18-21}$

(18) Zhu, J.; Withers, S. G.; Reichardt, P. B.; Treadwell, E.; Clausen, T. P. Biochem. J. 1998, 332, 367-371.

(19) Halazy, S.; Berges, V.; Ehrhard, A.; Danzin, C. Bioorg. Chem. 1990, $18,330-344$.

(20) McCarter, J. D.; Withers, S. G. J. Am. Chem. Soc. 1996, 118, 241242 .

(21) Azoulay, M.; Chalard, F.; Cesson, J.-P.; Florent, J.-C.; Monneret, C. Carbohydr. Res. 2001, 332, 151-156.

\section{Coomassie Streptavidin}

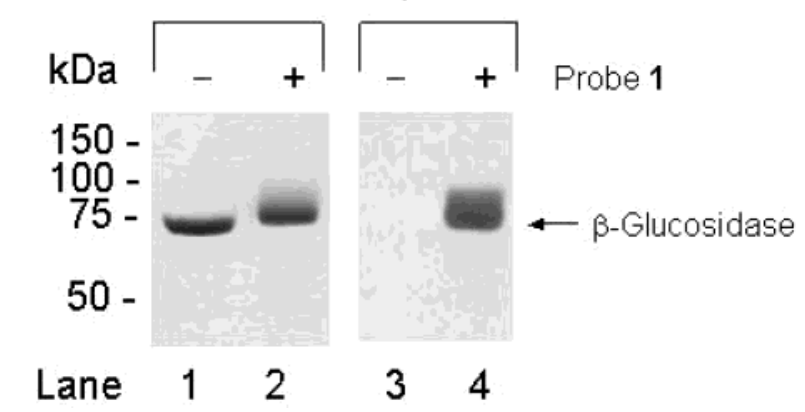

Figure 2. Gels showing the $\beta$-glucosidase-mediated activation of the probe 1 , resulting in biontinylated modification of the enzyme. Reaction mixtures were separated by $8 \%$ SDS-PAGE. Coomassie blue-stained (left) and ECL-developed (right) gels are presented.

they have rarely been utilized as labeling probes. Activity probes can selectively and covalently label a target protein without reducing activity when they modify nonessential residues. Besides, an activity probe could serve in a wide range of applications depending on the property of the reporter groups. To achieve these goals, it would need additional tests and possible alterations to function well in various systems. For example, the prototype probe developed in this study is ready to use for rapid screening of glycosidases from numerous microbial sources. However, it produced cross-labeling when activated in the presence of a mixture of proteins in a preliminary study (data not shown) and might not be suitable for proteomics applications in its current format. This result is different from that of tyrosine phosphatase probe, ${ }^{4}$ in which case no cross-labeling was observed, indicating that the structure of the biocatalysts also plays an important role in determining the performance of this type of probe.

In summary, we have accomplished a new synthetic route for the activity probe of $\beta$-glucosidase. Benzyl $p$-hydroxyphenylacetate was used as the starting point to overcome the difficulties in the glycosidation step. Benzylic functionalization for the trapping device was successfully introduced at later stages. Probe $\mathbf{1}$ developed in this study was able to label a model $\beta$-glucosidase. Although only a single probe was prepared and examined in this study, its cassette-like design provides great flexibility for future alterations. It not only allows various sugar units and different anomeric configurations to be constructed in the future to offer important intermediate 9 but it could also incorporate a large number of linker/reporter combinations to meet demands in various applications. We are currently studying the effect of a trapping device ${ }^{2,22}$ together with the linker/reporter combination, including the length and hydrophilic/hydrophobic properties, to improve their performance in proteomics applications. These results will be reported in due course.

(22) Ichikawa, M.; Ichikawa, Y. Bioorg. Med. Chem. Lett. 2001, 11 1769-1773 
Acknowledgment. This work was supported by the National Science Council (NSC 91-3112-B-002-013) (to L.-C.L.). We also thank Professor Jing-Jer Lin for helpful discussions.

Supporting Information Available: Experimental procedures and characterization, including copies of ${ }^{1} \mathrm{H}$ and
${ }^{13} \mathrm{C}$ NMR, for compounds $\mathbf{1}, \mathbf{4 - 9}$, and $\mathbf{1 1}$ as well as conditions for the labeling of $\beta$-glucosidase with probe $\mathbf{1}$. This material is available free of charge via the Internet at http://pubs.acs.org.

OL0265315 